\title{
Abundances ratios in star-forming galaxies: clues to galaxy evolution
}

\author{
Thierry Contini ${ }^{1}$, Marie-A. Treyer ${ }^{2}$, and Mustapha Mouhcine ${ }^{3}$ \\ ${ }^{1}$ Laboratoire d'Astrophysique, Observatoire Midi-Pyrenees, \\ UMR 5572, 14 avenue E. Belin, F-31400 Toulouse, la France \\ ${ }^{2}$ Laboratoire d'Astrophysique de Marseille, - UMR 6110 \\ Traverse du Siphon, BP 8, F-13376 Marseille Cedex 12, la France \\ ${ }^{3}$ University of California, Los Angeles, CA 90095-1567, USA
}

\begin{abstract}
We show in this contribution that detailed studies of chemical abundances in UV-selected, $\mathrm{H}$ II and starburst nucleus galaxies, together with the development of new chemical evolution models, put strong constraints on the evolutionary stage of starburst galaxies in terms of star formation history. Although the samples of high-redshift galaxies are still too small for statistical studies, the preliminary results give insight into the nature and evolution of distant star-forming objects and their link with present-day galaxies.
\end{abstract}

Tracing the star-formation history (SFH) of galaxies is essential for understanding galaxy formation and evolution. The chemical properties of galaxies are closely related to their $\mathrm{SFH}$, and can be considered as fossil records, enabling us to track the galaxy formation history down to the present. There is a lot of observational evidence suggesting that the SFH of galaxies has not been monotonic with time, but exhibits instead significant fluctuations. Examining the chemical evolution and physical nature of star-forming galaxies over a range of redshifts will shed light on this issue. Emission lines from $\mathrm{H}$ II regions have long been the primary means of chemical diagnosis in local galaxies, but this method has only recently been applied to galaxies at cosmological distances following the advent of infrared spectrographs on $8-10 \mathrm{~m}$ class telescopes (e.g., Pettini et al. 2001; Lemoine-Busserolle et al. in these Proceedings).

Contini et al. (2002) recently derived the chemical properties of a intermediate redshift $(0<z<0.4)$ sample of UV-selected galaxies. These objects are found to be intermediate between low-mass, metal-poor H II galaxies and more massive, metal-rich starburst nucleus galaxies, spanning a wide range of oxygen abundances, from $\sim 0.1$ to $1 \mathrm{Z}_{\odot}$. The behavior of these starburst galaxies in the $\mathrm{N} / \mathrm{O}$ vs. $\mathrm{O} / \mathrm{H}$ relation (see Figure 1 ) has been investigated in order to probe their physical nature and SFH. At a given metallicity, the majority of UV-selected galaxies has low N/O abundance ratios. The most plausible interpretation could be that UV galaxies are observed at a special stage in their evolution, following a powerful starburst that enriched their ISM in oxygen. A natural explanation for the variation of $\mathrm{N} / \mathrm{O}$ at constant metallicity might be a significant time delay between the release, into the ISM, of oxygen by massive, short-lived stars and that of nitrogen produced in low-mass longer-lived stars. Extensive chemical 

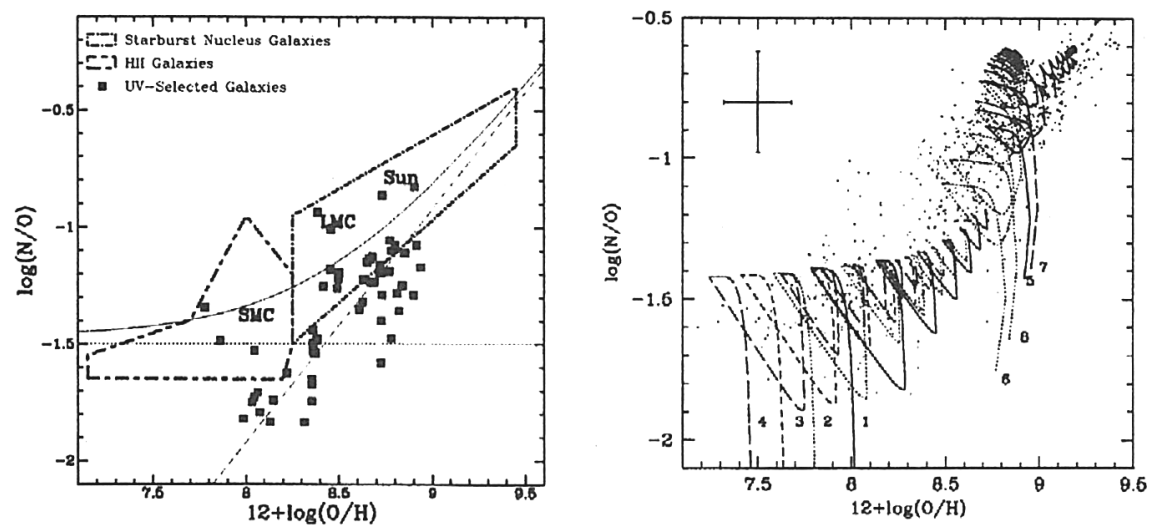

Figure 1. Left: $\mathrm{N} / \mathrm{O}$ vs. $12+\log (\mathrm{O} / \mathrm{H})$ for the UV-selected galaxies (squares) and two comparison samples of nearby star-forming galaxies: starburst nucleus galaxies and $\mathrm{H}$ II galaxies (see Contini et al. 2002 for references). Right: Predictions of chemical evolution models assuming bursting SF scenario (model parameters are listed in Mouhcine \& Contini 2002).

evolution model computations (see Figure 1 and Mouhcine \& Contini 2002 for details) show that no possible combination of the model parameters (i.e., burst duration, interburst period, SF efficiency, gas accretion timescale, etc.) is able to account for the observed spread in the $\mathrm{N} / \mathrm{O}$ vs. $\mathrm{O} / \mathrm{H}$ diagram for the whole sample of galaxies. They found that metal-rich spiral galaxies differ from metalpoor ones by a higher SF efficiency and starburst frequency. Low-mass galaxies experienced a few bursts of SF, whereas massive spiral galaxies experienced numerous and extended powerful starbursts.

Although the samples of high-redshift galaxies with accurate determination of their physical properties are still too small to derive firm conclusions on the link between distant objects and present-day galaxies, the preliminary results obtained so far (e.g., Pettini et al. 1998, 2001; Kobulnicky \& Koo 2000; Hammer et al. 2001; Contini et al. 2002; Lemoine-Busserolle et al. in these Proceedings) give new insight into the nature and evolution of distant star-forming galaxies. No doubt that the next large-scale spectroscopic surveys on $10 \mathrm{~m}$ class telescopes (e.g., VIRMOS on VLT and COSMOS/EMIR on GranTeCan) will shed light on these fundamental issues by producing statistically significant samples of galaxies over a large range of redshifts.

\section{References}

Contini, T., Treyer, M.-A., Sullivan, M., Ellis, R.S. 2002, MNRAS 330, 75

Hammer, F.; Gruel, N., Thuan, T.X., et al. 2001, ApJ 550, 570

Kobulnicky, H.A., Koo, D.C. 2000, ApJ 545, 712

Mouhcine, M., Contini, T. 2002, A\&A 389, 106

Pettini, M., Kellogg, M., Steidel, C.C., et al. 1998, ApJ 508, 539

Pettini, M., Shapley, A. E., Steidel, C.C., et al. 2001, ApJ 554, 981 